\title{
Distúrbios Metabólicos e Adiposidade em uma População Rural
}

\begin{abstract}
RESUMO
O objetivo deste estudo transversal foi investigar a prevalência de sobrepeso e adiposidade central (AC) e suas relações com alterações no metabolismo de lipídeos, glicose e níveis pressóricos em uma amostra de 287 indivíduos adultos residentes em uma comunidade rural do Estado de Minas Gerais. Foram realizadas comparações de médias dos indicadores metabólicos: lipoproteínas, glicose e níveis pressóricos, segundo categorias de sobrepeso e $\mathrm{AC}$, usando análise de variância e teste de Tukey. A prevalência de sobrepeso foi de $24,8 \%$ ( $37,4 \%$ no sexo feminino; $11,5 \%$ no sexo masculino). A AC esteve presente em $28,1 \%$ das pessoas $(50,3 \%$ no sexo feminino; $4,3 \%$ no sexo masculino). Indivíduos com AC apresentaram maiores valores médios de pressão arterial (HA), colesterol total, LDL, triglicérides e glicemia de jejum; e menores valores médios de HDL. Foram verificadas associações entre AC e HA, dislipidemia e hiperglicemia. Associações significativas entre sobrepeso e $\mathrm{HA}$, dislipidemia e hiperglicemia também foram evidenciadas. Esses resultados confirmam o potencial efeito das alterações na composição corporal, principalmente em nível central, sobre o metabolismo lipídico e de glicose, e também sobre o aumento dos níveis pressóricos em populações da área rural. (Arq Bras Endocrinol Metab 2008; 52/3:489-498)
\end{abstract}

Descritores: Adiposidade; Sobrepeso; Dislipidemia; Hipertensão; Hiperglicemia; População rural

\section{ABSTRACT}

\section{Metabolic Disorders and Adiposity in a Rural Population.}

The aim of this cross-sectional study was to verify the prevalence of overweight and central adiposity (CA) in a sample of 287 adult subjects that lived in a rural community of Minas Gerais State. Means lipids, lipoproteins, glucose and blood pressure levels were compared according adiposity categories using One-way ANOVA and Tukey tests. Prevalence of overweight was $24.8 \%(37.4 \%$ for female; $11.5 \%$ for male). CA was verified in $28.1 \%$ of the individuals ( $50.3 \%$ for female; $4.3 \%$ for male). The associations between CA and overweight with the metabolic disorders: arterial hypertension (AH), dyslipidemia and hyperglycemia were estimated. Subjects with CA presented higher mean values of blood pressure, total cholesterol, LDL, triglycerides, fasting glucose, and lower mean values of HDL. CA was associated with $\mathrm{AH}$, dyslipidemia and hyperglycemia. Associations between overweight and $\mathrm{AH}$, dyslipidemia and hyperglycemia were also verified. These results confirm the potential effect of body composition shifting, especially at the abdominal level, on lipids, glucose metabolism and on blood pressure levels in rural populations. (Arq Bras Endocrinol Metab 2008; 52/3:489-498)

Keywords: Adiposity; Overweight; Dyslipidemia; Hypertension; Hyperglycemia; Rural population

\section{artigo original}

\author{
Daniele A. Silva \\ Mariana S. Felisbino-Mendes \\ Adriano M. Pimenta \\ AndREa GazzInelLI \\ Gilberto KaC \\ Gustavo Velásquez-Meléndez
}

Departamento de Enfermagem Materno Infantil e Saúde Pública (EMI) da Universidade Federal de Minas Gerais (UFMG), Belo Horizonte, MG, Brasil (DAS, MSFM, AMP, AG, GVM); Instituto de Nutrição Josué de Castro do Departamento de Nutrição Social e Aplicada (DNSA) da Universidade Federal do Rio de Janeiro (UFRJ), RJ, Brasil (GK).

Recebido em 26/01/2007 Aceito em 05/12/2007 


\section{INTRODUÇÃO}

OBESIDADE ATUALMENTE É um grave problema de saúde pública em nível mundial e tem sido freqüentemente associada à melhora do acesso a alimentos de alta densidade energética e ao estilo de vida sedentário, hábitos de vida mais modernos, que surgiram a partir do desenvolvimento tecnológico e social das populações, principalmente as ocidentais $(1,2)$.

A obesidade se caracteriza como um problema na medida em que o excesso de peso e a gordura abdominal se associam fortemente a anormalidades metabólicas (1). Tal associação promove um crescente risco de desenvolvimento de doenças cardiovasculares (DCV), as quais representaram no Brasil, em 2002, 28\% das mortes por causas básicas conhecidas, além de serem consideradas o principal motivo de hospitalização no serviço público de saúde (3).

Nesse contexto, a relevância da obesidade é evidenciada em diversos estudos que atualmente entendem as células adiposas como componentes de um órgão com atividade endócrina e metabólica e não apenas como integrantes de um tecido de armazenamento de energia e sustentação (4). Assim, alguns autores demonstraram que a gordura localizada em nível abdominal (ou obesidade visceral) associa-se mais fortemente a distúrbios metabólicos do que a gordura acumulada em outras partes do corpo (4-9).

Indivíduos com obesidade visceral apresentam uma série de distúrbios que produzem alterações no metabolismo de lipídeos, glicose e insulina. Esses indivíduos apresentam um estado de hiperlipidemia pós-prandial em virtude de superprodução hepática de lipoproteínas ricas em triglicérides, aumentando a concentração delas na circulação porta hepática, e promovendo uma espécie de competição com os quilomícrons advindos da dieta (7). Em relação à glicose sanguínea, elevados níveis de glicemia não só caracterizam o diabetes como também aumentam os riscos de retino, nefro e neuropatias, além de representarem fator de risco para o desenvolvimento de arteriosclerose (7).

Alterações no perfil metabólico de lipoproteínas podem também ser esperadas como resultado de dietas inadequadas e sedentarismo (10).

O processo de transição nutricional tem sido observado em populações de regiões metropolitanas desde os anos de 1970 (11), entretanto, temos poucas evidências que essas mudanças tenham ocorrido também em popu- lações de áreas rurais do Brasil. O padrão de composição e distribuição corporal, assim como o perfil de lipídeos, podem ser importantes indicadores dessas mudanças. $\mathrm{O}$ presente estudo tem como objetivo estimar as prevalências de sobrepeso e adiposidade central (AC), bem como as relações desses indicadores com o perfil lipídico, glicêmico e hipertensão arterial (HA) em população da área rural de Minas Gerais.

\section{MATERIAL E MÉTODOS}

Um estudo epidemiológico de base populacional e delineamento transversal foi desenvolvido na comunidade de Virgem das Graças, área rural do município de Ponto dos Volantes, Vale do Jequitinhonha, Minas Gerais, Brasil. Essa comunidade foi escolhida porque nela era desenvolvido um projeto interinstitucional, $\mathrm{e}$ essa população estava previamente cadastrada por meio de censo (12).

O número de pessoas adultas cadastradas pelo projeto anteriormente mencionado ( $\geq 18$ anos de idade) era de 351 indivíduos. Desse total foram excluídos 64; sete por estarem grávidas, três por motivos médico-cirúrgicos, 20 por estarem viajando, 11 por terem migrado para outras áreas e 23 por não-adesão à participação no estudo. Portanto, a população de estudo foi composta por 287 pessoas, 139 mulheres e 148 homens. O trabalho de campo foi desenvolvido por seis entrevistadores treinados. Um questionário estruturado com perguntas sobre sexo, idade, cor de pele, estado marital, escolaridade e história obstétrica, para as mulheres foi aplicado por meio de entrevista face a face. Também foi realizado um exame clínico, no qual foram aferidas algumas medidas antropométricas, segundo recomendações (13). Todas as medidas foram realizadas três vezes, sendo o valor final determinado pela média entre elas.

As medidas antropométricas realizadas foram peso, estatura e circunferências da cintura e do quadril. Para a medida do peso corporal foi utilizada uma balança digital (Modelo PL 150, Filizzola Ltda.) com aproximação de $0,1 \mathrm{~kg}$. A estatura foi aferida com o indivíduo em pé, ereto com a cabeça no plano de Frankfurt, com aproximação de $0,1 \mathrm{~cm}$ e usando uma fita inextensível. As circunferências corporais (cintura e quadril) foram medidas até o milímetro mais próximo usando uma fita inelástica com o sujeito em pé, ereto, com o abdome relaxado e desnudo, braços sol- 
tos e os pés juntos. Para a medida da circunferência da cintura, a fita foi posicionada no ponto médio entre a parte inferior da costela e a parte superior da crista ilíaca. A circunferência do quadril foi medida na área de maior protuberância das nádegas.

A partir do peso e da estatura, foi calculado o índice de massa corporal $\left[\mathrm{IMC}=\right.$ peso $(\mathrm{kg}) /$ estatura $\left.(\mathrm{m})^{2}\right] . A$ razão circunferência da cintura/circunferência do quadril (RCQ) foi calculada usando as circunferências da cintura e do quadril.

Foram colhidas amostras de aproximadamente 5 $\mathrm{ml}$ de sangue, obtidas por meio de punção venosa, com o paciente em jejum de 12 horas. A coleta e a centrifugação das amostras sanguíneas ocorreram no local da pesquisa. O soro e o plasma de cada indivíduo foram separados em tubos de ensaios devidamente identificados e enviados ao laboratório no mesmo dia da coleta, seguindo especificações técnicas recomendadas.

No laboratório, as amostras de soro e plasma foram armazenadas em um refrigerador a $4{ }^{\circ} \mathrm{C}$ e analisadas usando o analisador Cobas Mira Plus (Roche), que era regularmente calibrado. As concentrações do CT, triglicérides e glicose foram determinadas usando um teste enzimático colorimétrico. A concentração da lipoproteína de alta densidade (HDL) também foi medida por um teste enzimático colorimétrico, após precipitação das frações LDL (lipoproteína de baixa densidade) e lipoproteína de muito baixa densidade (VLDL) pelo ácido fosfotúngstico e cloreto de magnésio. Os níveis de LDL foram calculados por aplicação da equação de Friedwald: $\mathrm{LDL}=\mathrm{CT}-(\mathrm{HDL}+\mathrm{TG} / 5)$, em que CT é o colesterol total e TG, triglicérides.

As variáveis neste estudo foram categorizadas da seguinte forma: idade (18-29, 30-39, 40-49, 50-59 e $\geq$ 60 anos); escolaridade (analfabeto, $1-4 \geq 5$ anos); estado marital (casada, em união, separada, viúva); cor de pele (branca, parda ou preta); IMC $\geq 25 \mathrm{~kg} / \mathrm{m}^{2}$ ( $\mathrm{sim}$, não) (14). A AC foi definida usando a RCQ (RCQ $\geq$ 0,85 para mulheres e RCQ $\geq 1,00$ para homens). As dislipidemias foram definidas usando as III Diretrizes sobre Dislipidemias e Prevenção da Arteriosclerose (15): CT $\geq 200 \mathrm{mg} / \mathrm{dL}$, LDL-C $\geq 130 \mathrm{mg} / \mathrm{dL}$, HDL-C $<40 \mathrm{mg} / \mathrm{dL}, \mathrm{TG} \geq 200 \mathrm{mg} / \mathrm{dL}$ e a hiperglicemia, segundo a American Diabetes Association (16): glicemia de jejum $\geq 100 \mathrm{mg} / \mathrm{dL}$.

Consideraram-se indivíduos com HA aqueles que apresentaram valores de pressão arterial sistólica (PAS) $\geq 140 \mathrm{mmHg}$ e pressão arterial diastólica $(\mathrm{PAD}) \geq 90$ $\mathrm{mmHg}$ ou àquelas que faziam uso de medicação antihipertensiva (17). Para tanto, a pressão arterial foi aferida por três vezes e dos resultados foi obtida uma média simples. Para as análises dos níveis pressóricos foi usada a média ponderada das duas pressões usando a fórmula $(\mathrm{PAM}=\mathrm{PAS}+2 \mathrm{PSD}) / 2$.

A síndrome metabólica (SM) foi definida segundo o critério diagnóstico proposto pela National Cholesterol Education Program's Adult Treatment Panel III (NCEP) (18).

As análises estatísticas foram realizadas utilizandose o programa STATA versão 9.1. Inicialmente foram realizadas comparações de médias dos indicadores metabólicos: lipoproteínas, glicose e níveis pressóricos, segundo categorias de sobrepeso e $\mathrm{AC}$ ( e $\mathrm{AC}=$ não, sobrepeso $=$ não e $\mathrm{AC}=\operatorname{sim}$, sobrepeso $=$ sim e $\mathrm{AC}=$ não, sobrepeso $=\operatorname{sim}$ e $\mathrm{AC}=\operatorname{sim})$ usando análise de variância (Anova) e teste de Tukey. As variáveis que não apresentaram distribuição normal foram normalizadas utilizando-se a função logarítmica e a análise foi refeita. Como não houve diferenças entre os resultados das análises, optou-se por trabalhar com as variáveis não transformadas.

Foram testadas ainda as associações entre o sobrepeso, a AC e as variáveis indicadoras de distúrbios metabólicos: hipertensão, dislipidemias e hiperglicemia, usando como medida de associação a razão de prevalência com a utilização da regressão de Poisson. As razões de prevalência foram ajustadas por idade, escolaridade, tabagismo e consumo de álcool. Em todas as análises adotou-se nível de significância de $5 \%(\mathrm{p}<0,05)$.

A presente pesquisa foi aprovada pelo Comitê de Ética em Pesquisa da Universidade Federal de Minas Gerais (UFMG), e autorizações por escrito foram obtidas de todas as participantes do estudo.

\section{RESULTADOS}

A distribuição da população de Virgem das Graças é relativamente homogênea no que diz respeito ao sexo: $51,6 \%(n=148)$ eram mulheres e $48,4 \%(n=139)$ eram homens. Observou-se que as mulheres eram viúvas com maior freqüência e fumavam e bebiam em menor proporção do que os homens $(\mathrm{p}<0,001)$. No que diz respeito à escolaridade, $37,7 \%$ da população não tiveram educação formal (Tabela 1 ). 
Tabela 1. Características demográfica, socioeconômicas e do estilo de vida segundo o sexo. Virgem das Graças, MG - 2004.

\begin{tabular}{|c|c|c|c|c|}
\hline \multirow{3}{*}{$\begin{array}{l}\text { Características da amostra } \\
\text { Faixa etária (anos) }\end{array}$} & \multicolumn{2}{|c|}{ Sexo } & \multirow{3}{*}{ Total n (\%) } & \multirow{3}{*}{$\begin{array}{c}\text { p-valor* } \\
0,901\end{array}$} \\
\hline & Masculino n (\%) & Feminino $n(\%)$ & & \\
\hline & & & & \\
\hline $18-29$ & $34(24,5)$ & $42(28,4)$ & $76(26,4)$ & \\
\hline $30-39$ & $27(19,4)$ & $26(17,6)$ & $53(18,5)$ & \\
\hline $40-49$ & $22(15,8)$ & $20(13,5)$ & $42(14,7)$ & \\
\hline $50-59$ & $24(17,3)$ & $23(15,5)$ & $47(16,4)$ & \\
\hline$\geq 60$ & $32(23,0)$ & $37(25,0)$ & $69(24,0)$ & \\
\hline Cor de pele & & & & 0,170 \\
\hline Branca & $50(36,0)$ & $65(43,9)$ & $115(40,1)$ & \\
\hline Parda/negra & $89(64,0)$ & $83(56,1)$ & $172(59,9)$ & \\
\hline Estado marital & & & & 0,060 \\
\hline Casado & $76(54,6)$ & $86(58,1)$ & $162(56,5)$ & \\
\hline Em união & $29(20,9)$ & $19(12,8)$ & $48(16,7)$ & \\
\hline Solteiro & $30(21,6)$ & $23(15,6)$ & $53(18,5)$ & \\
\hline Viúva/separado & $4(2,9)$ & $20(13,5)$ & $24(8,3)$ & \\
\hline Escolaridade (anos) & & & & 0,629 \\
\hline Analfabeto & $51(36,7)$ & $57(38,5)$ & $108(37,7)$ & \\
\hline $1-4$ & $58(41,7)$ & $54(36,5)$ & $112(39,0)$ & \\
\hline$\geq 5$ & $30(21,6)$ & $37(25,0)$ & $67(23,4)$ & \\
\hline Hábito de fumar & & & & $<0,001$ \\
\hline Não & $52(37,4)$ & $118(79,7)$ & $170(59,2)$ & \\
\hline Sim & $46(33,1)$ & $18(12,2)$ & $64(22,3)$ & \\
\hline Ex-fumante & $41(29,5)$ & $12(8,1)$ & $53(18,5)$ & \\
\hline Consumo de álcool & & & & $<0,001$ \\
\hline Sim & $40(28,8)$ & $13(8,8)$ & $53(18,5)$ & \\
\hline Não & $99(71,2)$ & $135(91,2)$ & $234(81,5)$ & \\
\hline
\end{tabular}

O sobrepeso e a obesidade estiveram presentes em $18,6 \%$ e $6,2 \%$ das pessoas, respectivamente. Entretanto, ao se comparar essas prevalências em relação ao sexo, observou-se uma diferença significativa $(\mathrm{p}<0,05)$, visto que tais condições eram mais freqüientes nas mulheres, $26,5 \%$ e $10,1 \%$, respectivamente. A prevalência de hipertrigliceridemia foi de $8,3 \%$ e também se diferenciou significativamente em relação ao sexo, acometendo $11,9 \%$ das mulheres e $4,2 \%$ dos homens (Tabela 2 ). A freqüência de AC foi de $28,1 \%$, sendo muito elevada entre as mulheres $(50,3 \%)$ (Tabela 2$)$.

Quase metade das pessoas apresentava níveis altos de CT e de LDL, acima de $130 \mathrm{mg} / \mathrm{dL}$. A freqüência de indivíduos com hiperglicemia foi de $15,4 \%$, e nesse contingente estavam incluídos os pacientes sabidamen- 


\begin{tabular}{|c|c|c|c|c|}
\hline \multirow{3}{*}{$\begin{array}{l}\text { Características da amostra } \\
\operatorname{IMC}^{1}\left(\mathrm{~kg} / \mathrm{m}^{2}\right)\end{array}$} & \multicolumn{2}{|c|}{ Sexo } & \multirow[b]{2}{*}{ Total n (\%) } & \multirow[b]{2}{*}{ p-valor* } \\
\hline & Masculino $n(\%)$ & Feminino $\mathrm{n}(\%)$ & & \\
\hline & & & & $<0,001$ \\
\hline Baixo peso $(<18,5)$ & $8(5,8)$ & $7(4,8)$ & $15(5,2)$ & \\
\hline Normal $(18,5-24,9)$ & $115(82,7)$ & $85(57,8)$ & $200(69,9)$ & \\
\hline Sobrepeso $(25,0-29,9)$ & $14(10,1)$ & $39(26,5)$ & $53(18,6)$ & \\
\hline Obeso $(\geq 30,0)$ & $2(1,4)$ & $16(10,9)$ & $18(6,2)$ & \\
\hline$A C^{2}$ & & & & $<0,001$ \\
\hline Não & $132(95,7)$ & $73(49,7)$ & $205(71,9)$ & \\
\hline Sim & $6(4,3)$ & $74(50,3)$ & $80(28,1)$ & \\
\hline Colesterol total (mg/dL) & & & & 0,994 \\
\hline$<200$ & $62(52,5)$ & $71(52,6)$ & $133(52,6)$ & \\
\hline$\geq 200$ & $56(47,5)$ & $64(47,4)$ & $120(47,4)$ & \\
\hline $\mathrm{LDL}^{3}$ (mg/dL) & & & & 0,206 \\
\hline$<130$ & $70(59,8)$ & $73(54,0)$ & $143(56,7)$ & \\
\hline $130-159$ & $25(21,4)$ & $30(22,2)$ & $55(21,8)$ & \\
\hline$\geq 160$ & $22(18,8)$ & $32(23,7)$ & $54(21,4)$ & \\
\hline $\mathrm{HDL}^{4}(\mathrm{mg} / \mathrm{dL})$ & & & & 0,392 \\
\hline$\leq 40$ & $91(77,1)$ & $110(18,5)$ & $201(79,5)$ & \\
\hline$>40$ & $27(22,9)$ & $25(81,5)$ & $52(20,5)$ & \\
\hline Triglicérides (mg/dL) & & & & 0,029 \\
\hline$<200$ & $113(95,8)$ & $119(88,1)$ & $232(91,7)$ & \\
\hline$\geq 200$ & $5(4,2)$ & $16(11,9)$ & $21(8,3)$ & \\
\hline Glicemia de jejum (mg/dL) & & & & 0,673 \\
\hline$<100$ & $101(85,6)$ & $113(83,7)$ & $214(84,6)$ & \\
\hline $100-125$ & $14(11,9)$ & $20(14,8)$ & $34(13,4)$ & \\
\hline$\geq 126$ & $3(2,5)$ & $2(1,5)$ & $5(2,0)$ & \\
\hline Hipertensão ${ }^{6}$ & & & & 0,573 \\
\hline Sim & $63(45,3)$ & $72(48,6)$ & $135(47,0)$ & \\
\hline Não & $76(54,7)$ & $76(51,4)$ & $152(53,0)$ & \\
\hline Dislipidemia ${ }^{7}$ & & & & 0,848 \\
\hline Sim & $69(59,0)$ & $78(57,8)$ & $147(58,4)$ & \\
\hline Não & $48(41,0)$ & $57(42,8)$ & $105(41,6)$ & \\
\hline Síndrome metabólica & & & & $<0,001$ \\
\hline Sim & $12(9,4)$ & $46(32,4)$ & $58(21,5)$ & \\
\hline Não & $116(90,6)$ & $96(67,6)$ & $212(78,5)$ & \\
\hline
\end{tabular}




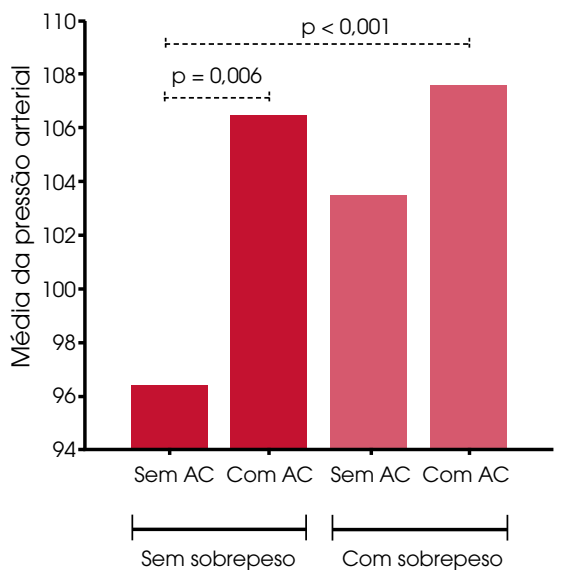

Figura 1A. Comparação das médias (valor de p, teste de Tukey) de pressão arterial média, segundo sobrepeso e AC na população rural de Virgem das Graças, MG, 2004.

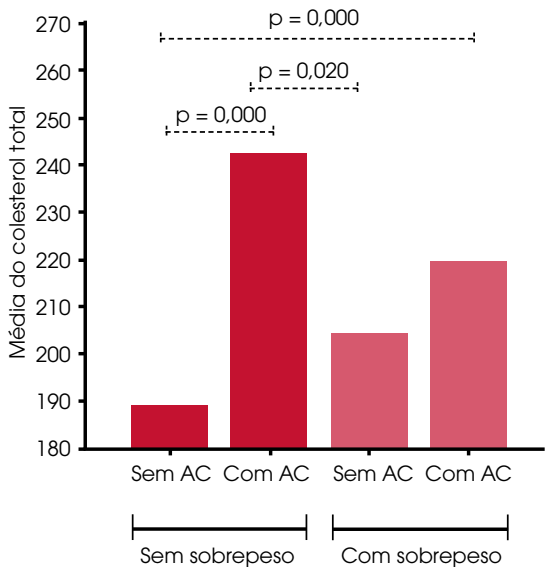

Figura 1B. Comparação das médias (valor de p, teste de Tukey) do CT, segundo sobrepeso e AC na população rural de Virgem das Graças - MG, 2004.

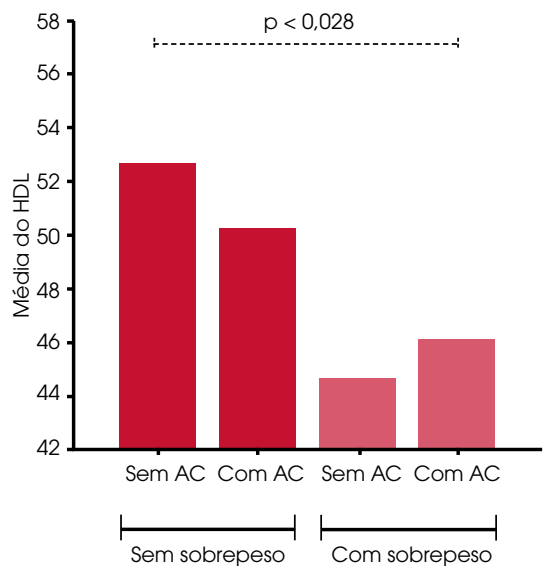

Figura 1C. Comparação das médias (valor de p, teste de Tukey) de HDL, segundo sobrepeso e AC na população rural de Virgem das Graças - MG, 2004.

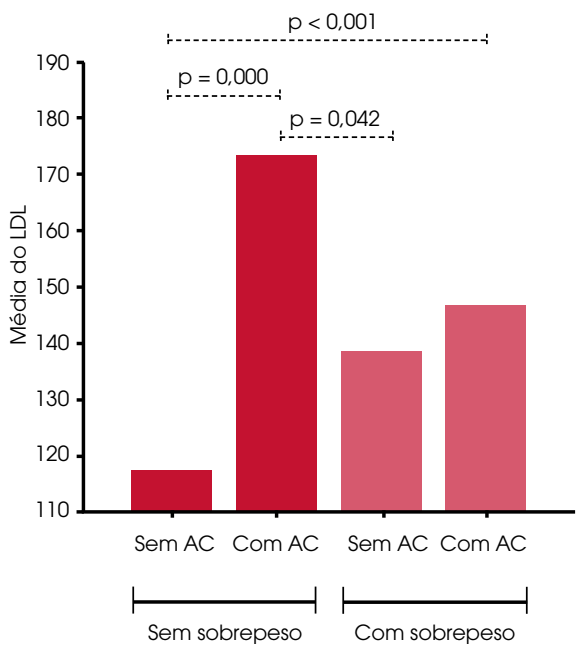

Figura 1D. Comparação das médias (valor de $\mathrm{p}$, teste de Tukey) de LDL, segundo sobrepeso e AC na população rural de Virgem das Graças - MG, 2004.

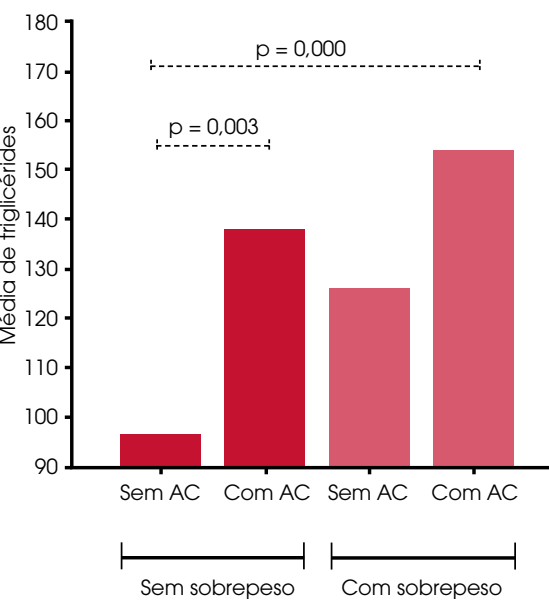

Figura 1E. Comparação das médias (valor de $\mathrm{p}$, teste de Tukey) de TG, segundo sobrepeso e AC na população rural de Virgem das Graças - MG, 2004.

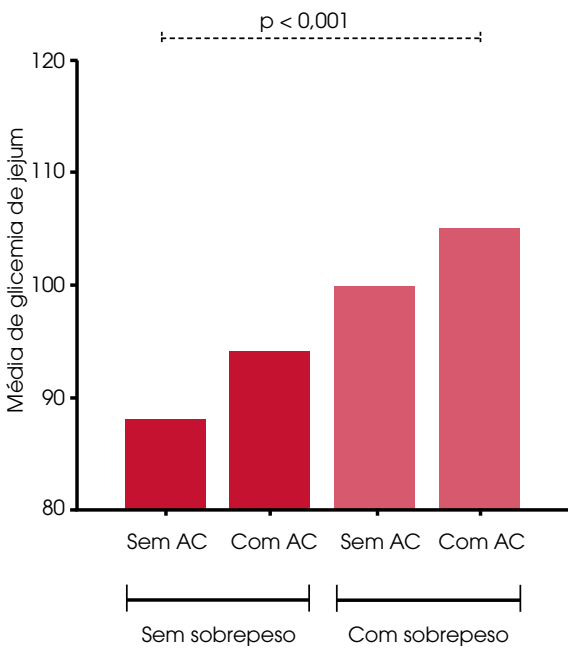

Figura 1F. Comparação das médias (valor de p, teste de Tukey) de glicemia de jejum, segundo sobrepeso e AC na população rural de Virgem das Graças - MG, 2004. te diabéticos (Tabela 2). A prevalência de diabetes nesta população foi de 3,4\% (dados não apresentados).

Em relação níveis de HDL e TG, observa-se que, respectivamente, $20,5 \%$ e $8,3 \%$ da população possuíam esses componentes bioquímicos alterados. As prevalên- cias de HA e dislipidemia foram altas na população estudada, respectivamente, $47,0 \%$ e $58,4 \%$ (Tabela 2 ).

A prevalência da SM foi de $21,5 \%$, sendo estatisticamente $(\mathrm{p}<0,001)$ maior nas mulheres $(32,4 \%)$ do que nos homens $(9,4 \%)$ (Tabela 2$)$. 
Na Figura 1 são apresentadas comparações das médias de algumas variáveis, mostrando que os indivíduos com AC apresentaram valores médios maiores de pressão arterial (PA), CT, LDL, TG e glicemia de jejum e valores menores de HDL. Na Tabela 3 observou-se que o sobrepeso esteve associado à hipertensão $(\mathrm{RP}=1,69$; IC 95\%: 1,34 2,13), dislipidemia ( $\mathrm{RP}=1,46$; IC 95\%: $1,20-1,78)$ e hiperglicemia ( $R P=2,79$; IC 95\%: 1,59-4,89). Simultaneamente foi mostrada associação entre AC e hipertensão ( $R P=1,68$; IC 95\%: 1,33-2,11), dislipidemia $(R P=1,62$; IC 95\%: 1,34-1,95) e hiperglicemia $(\mathrm{RP}=2,52$; IC 95\%: $1,43-4,44)$.

\section{DIscussÃo}

O presente estudo mostra que as prevalências de sobrepeso e AC em mulheres residentes em área rural foram similares às encontradas em populações de áreas metropolitanas do sudeste do Brasil (19). Adicionalmente verificaram-se altas freqüências de dislipidemia e HA, sem diferenças entre os sexos. Nota-se apenas menores prevalências de valores inadequados de TG nos homens quando comparadas às mulheres. Os níveis glicêmicos foram similares em ambos os sexos.

Apesar de o presente estudo não ter avaliado o nível de atividade física, a maior prevalência de hipertrigliceridemia nas mulheres pode ser um indicativo de baixa atividade física nesse grupo. Como mostrado em outros estudos, o sedentarismo pode contribuir para o aumento dos níveis de TG (20). Estudo similar realizado em comunidade rural do Estado da Bahia mostrou inadequado perfil lipídico, baixos níveis de atividade física e alta freqüência de obesidade abdominal em pessoas do sexo feminino (21).

Em estudo recente realizado na população de Virgem das Graças foram observadas altas taxas de infestação parasitária por esquistossomose (22), que juntamente com as altas prevalências de obesidade nas mulheres, altas taxas de hipertensão e dislipidemia em ambos os sexos, caracterizariam esta população em processo inicial de transição epidemiológica e nutricional. Estudos em comunidades amazônicas do Brasil mostraram também um perfil de risco cardiovascular similar à população de área metropolitana dessa mesma região (23). Em um trabalho desenvolvido em uma comunidade rural do semi-árido baiano, também foram observadas altas prevalências de HA, dislipidemia, hiperglicemia e AC, porquanto essa última condição foi mais freqüente entre as mulheres (24).
Esses resultados podem ser em parte confirmatórios da tendência do aumento da obesidade associado à baixa renda, como observado também em populações pobres dos Estados Unidos (25) e populações rurais do México e da África do Sul $(26,27)$. Estudos em mulheres de áreas urbanas já mostraram que elas são mais propensas à obesidade, provavelmente, decorrentes de sua vulnerabilidade às mudanças em seus hábitos alimentares e estilo de vida, sendo os homens protegidos talvez por causa de atividades diárias que demandam mais esforço físico (28). O presente estudo também mostrou associação entre sobrepeso, AC e distúrbios metabólicos. Entretanto, é clara a importância da AC nesse contexto já que a sua relação com as alterações metabólicas é significante, inclusive na ausência de sobrepeso.

A relevância da obesidade como fator preditor de alterações metabólicas foi mostrada por Cercato e cols. (29) em estudo realizado em uma amostra de 1.213 brasileiros. Nesse estudo observou-se que o risco de desenvolver DCV cresceu com o aumento do IMC. Gus e cols. (30) demonstraram a importância da relação entre obesidade e alterações na PA em ambos os sexos, porém indicadores de AC associaram-se significantemente apenas entre as mulheres.

Bosello e Zamboni (31) também observaram associação entre a prevalência da AC com a presença de componentes da SM (resistência à insulina, alterações no metabolismo de insulina e lipídeos e aumento da PA), fato que reafirma a importância da relação entre gordura acumulada no abdome e distúrbios no metabolismo de lipídeos e carboidratos, além de alteração nos níveis pressóricos.

Krotkiewski e cols. (32) ressaltaram que, mesmo havendo diferenças na distribuição do tecido adiposo em homens e mulheres, a gordura acumulada na região abdominal torna os indivíduos de ambos os sexos mais suscetíveis a distúrbios no metabolismo de lipídeos e carboidratos.

Estudos observacionais realizados em diversas populações, inclusive do Brasil, têm mostrado estreita relação entre aumento da AC e da HA (8,33). Diversos métodos de estimação para avaliação da AC foram usados, como a circunferência da cintura, a relação cintura-quadril e até tomografia computadorizada (34).

Embora vários estudos mostrem uma consolidada relação entre a adiposidade regional, resistência à insulina, DCV, diabetes, dislipidemias e SM, os mecanismos biológicos dessas associações ainda são pouco claros. Alguns autores $(35,36)$ têm sugerido que a gordura visceral é um tecido altamente lipolítico, liberando, as- 
Tabela 3. Razão de prevalência de hipertensão, dislipidemia e glicemia de jejum segundo sexo, sobrepeso e adiposidade central. Virgem das Graças, MG - 2004

\begin{tabular}{lccc}
\hline Hipertensão' & $\begin{array}{c}\text { Razão de } \\
\text { prevalência }\end{array}$ & IC 95\% & p-valor \\
\hline Sexo (masculino/feminino) & 0,88 & $0,67-1,14$ & 0,324 \\
\hline Sobrepeso (sim/não) & 1,65 & $1,32-2,08$ & $<0,001$ \\
\hline Adiposidade central (sim/não) & 1,45 & $1,14-1,84$ & 0,002 \\
\hline Dislipidemia ${ }^{2}$ & & & 0,973 \\
\hline Sexo (masculino/feminino) & 0,99 & $0,78-1,26$ & $<0,001$ \\
\hline Sobrepeso (sim/não) & 1,45 & $1,19-1,76$ & 0,001 \\
\hline Adiposidade central (sim/não) & 1,43 & $1,16-1,77$ & 0,985 \\
\hline Glicemia de jejum $\geq 100$ mg/dL & & & 0,001 \\
\hline Sexo (masculino/feminino) & 0,99 & $0,55-1,81$ & 0,024 \\
\hline Sobrepeso (sim/não) & 2,58 & $1,49-4,49$ & $1,10-3,85$ \\
\hline Adiposidade central (sim/não) & 2,06 & & \\
\hline
\end{tabular}

' PAS Pressão arterial sistólica ( $\geq 140 \mathrm{mmHg}$ ) e/ou PAD ( $\geq 90 \mathrm{mmHg}) ;{ }^{2}$ Determinada a partir da presença de uma ou mais alterações nos níveis séricos de lipídeos. Todas as razões de prevalência foram ajustadas por idade, escolaridade, tabagismo e consumo de álcool, usando-se a técnica de regressão de Poisson.

sim, grande fluxo de ácidos graxos para o fígado, via sistema porta hepática, causando resistência hepática à insulina e aumento da liberação de VLDL e glicose pelo fígado. Essa hipótese ainda é contestada em relação à seqüência dos eventos relativos temporalidade da associação entre a resistência à insulina e o acúmulo de gordura visceral. Alternativamente considera-se possível que as duas condições possam ser resultados de um terceiro fator não identificado (37). Entretanto, trata-se de questão evidentemente não pode ser resolvida em um estudo com desenho transversal.

Uma limitação importante deste estudo está relacionada ao uso de medidas de adiposidade simples. Entretanto, é consenso a extrema dificuldade da aferição mais acurada da gordura visceral, já que requer métodos como a tomografia computadorizada ou a ressonância magnética, procedimentos que não são factíveis em estudos epidemiológicos.

A idade pode ser considerada uma variável que superestima as prevalências dos fatores de risco encontrados. Isso porque o processo natural de envelhecimento associado aos hábitos de vida, como má alimentação e sedentarismo, colaboram para alterações na composição corporal e no metabolismo de lipídeos e carboidratos. Neste estudo $24 \%$ da população era maior de 60 anos, situação esperada em populações rurais, uma vez que a migração dos indivíduos mais jovens é comum nessas populações. Tal fato sugere que a idade possa influenciar a relação entre composição corporal e distúrbios metabólicos quando se compara com populações urbanas.

Em síntese, foram mostradas prevalências significativamente maiores de obesidade global, AC e hipertrigliceridemia em mulheres quando comparados com os homens. Entretanto, os níveis de hiperglicemia e freqüências altas de hipertensão e dislipidemias não foram diferentes entre os sexos. As adiposidades global e central mostraram-se associadas a hipertensão, hiperglicemia e dislipidemias. Assim, os resultados deste trabalho confirmam o efeito potencial das alterações na composição corporal, principalmente o acúmulo de gordura no abdome, sobre os distúrbios do metabolismo lipídi- 
co e de glicose, além do aumento dos níveis pressóricos em populações pobres da área rural. Considerando que esses desfechos são importantes fatores de risco para morbimortalidade por DCV, eles poderão se constituir problemas de saúde significativos para as comunidades rurais no futuro. Portanto, a melhoria na qualidade e nos hábitos de vida nessas populações deveria constituir um dos objetos de trabalho das equipes multiprofissionais de toda a rede assistencial do Sistema Único de Saúde (SUS). É fundamental que esses serviços realizem atividades de promoção e educação em saúde, principalmente em áreas rurais, onde a atenção à saúde é muitas vezes ineficaz pela falta de recursos.

\section{AGRADECIMENTOS}

Fundação de Amparo à Pesquisa do Estado de Minas Gerais (Fapemig), National Institute of Health (NIHUSA), Fundação Oswaldo Cruz (Fiocruz), National Information Centre on Retirement Investments (Nicri), Conselho Nacional de Desenvolvimento Científico e Tecnológico (CNPq).

\section{REFERÊNCIAS}

1. Grundy SM. Obesity, metabolic syndrome and cardiovascular disease. J Clin Endocrinol Metab. 2004;89:2595-600.

2. Popkin BM. The nutrition transition and obesity in the developing world. J Nutr. 2001;131:871-73.

3. Ministério da Saúde - Datasus, Brasil. [acesso em 2005 May 14]. Disponível em: http://tabnet.datasus.gov.br/cgi/deftohtm. exe?sim/cnv/obtuf.def. (Acesso em 7 jan 2007)

4. Barroso SG, Abreu VG, Francischetti EA. A participação do tecido adiposo visceral na gênese da hipertensão e doença cardiovascular aterogênica. Um conceito emergente. Arq Bras Cardiol. 2002;78:618-30.

5. Lerario DD, Gimeno SG, Franco LJ, lunes M, Ferreira SR. Excesso de peso e gordura abdominal para a síndrome metabólica em nipo-brasileiros. Rev Saude Publica. 2002;36:4-11.

6. Nicklas BJ, Penninx BW, Cesari M, Kritchevsky SB, Newman $A B$, Kanaya $A M$, et al. Association of visceral adipose tissue with incident myocardial infarction in older men and women. Am J Epidemiol. 2004;160:741-9.

7. Scarsella C, Després JP. Tratamiento de la obesidad: necesidad de centrar la atención en los pacientes de alto riesgo caracterizados por la obesidad abdominal. Cad Saúde Pública. 2003;19:7-19.

8. Velásquez-Meléndez G, Kac G, Valente JG, Tavares R, Silva CQ, Garcia ES. Evaluation of waist circumference to predict general obesity and arterial hypertension in women in greater metropolitan Belo Horizonte, Brazil. Cad Saúde Pública. 2002;18:765-71.

9. Wannamethee SG, Shaper AG, Morris RW, Whincup PH. Measures of adiposity in the identification of metabolic abnormalities in elderly men. Am J Clin Nutr. 2005;81:1313-21.
10. World Health Organization - WHO. Food and agriculture organization: diet, nutrition and the prevention of chronic diseases. WHO, Technical Report Series, n. 916; 2003.

11. Mondini $L$, Monteiro $C A$. Mudanças no padrão de alimentação na população urbana brasileira (1962-1988). Rev Saúde Pública. 1997;28:433-9.

12. Kloos H, Passos LK, Loverde P, Oliveira RC, Gazzinelli A. Distribution and Schistosoma mansoni infection of Biomphalaria glabrata in different habitants in a rural area in the Jequitinhonha Valley, Minas Gerais, Brazil: environmental and epidemiological aspects. Mem Inst Oswaldo Cruz. 2004;99:673-81.

13. Lohman TG, Roche AF, Martorel A. Anthropometric standardization reference manual. Champaign: Human Kinetics Books, 1988.

14. World Health Organization - WHO. Physical status: the use and interpretation of antropometry. WHO, Technical Report Series, n. 854; 1995.

15. Departamento de Arteriosclerose da Sociedade Brasileira de Cardiologia. III Diretrizes sobre Dislipidemias de Prevenção de Arteriosclerose. Arq Bras Cardiol. 2001;77:4-8.

16. Genuth S, Alberti KG, Bennett P, Buse J, Defronzo R, Kahn R, et al. Follow-up report on the diagnosis of diabetes mellitus. Diabetes Care. 2003;26:3160-67.

17. Joint National Committee - JNC. The sixth report of the Joint National Committee on detection, evaluation, treatment of high blood pressure. Arch Intern Medicine. 1997;157:2413-46.

18. Grundy SM, Cleeman JI, Daniels SR, Donato KA, Eckel RH, Franklin BA, et al. Diagnosis and management of the metabolic syndrome: an American Heart Association/ National Heart, Lung, and Blood Institute Scientific Statement. Circulation. 2005;112:2735-52.

19. Velasquez-Melendez G, Pimenta AM, Kac G. Epidemiologia do sobrepeso e da obesidade e seus fatores determinantes em Belo Horizonte (MG), Brasil: estudo transversal de base populacional. Rev Panam Salud Publica. 2004;16:308-14.

20. Després JP, Lamarche B. Low-intensity endurance exercise training, plasma lipoproteins and the risk of coronary heart disease. J Intern Med. 1994;236:7-22.

21. Matos AC, Ladeia AM. Assessment of cardiovascular risk factors in a rural community in the Brazilian state of Bahia. Arq Bras Cardiol. 2003;81:297-302.

22. Gazzinelli A, Velasquez-Melendez G, Crawford SB, LoVerde PT, Correa-Oliveira R, Kloos $\mathrm{H}$. Socioeconomic determinants of schistosomiasis in a poor rural area in Brazil. Acta Trop. 2006;99:260-71.

23. Feio CM, Fonseca SA, Rego SS, Feio MN, Elias MC, Costa EA, et al. Lipid profile and cardiovascular risk in two Amazonian populations. Arq Bras Cardiol. 2003;81:596-9.

24. Oliveira EN, Souza MLA, Lima MDA. Prevalência de síndrome metabólica em uma área rural do semi-árido baiano. Arq Bras Endocrinol Metab. 2006;50:456-65.

25. Bruce MA, Sims M, Miller S, Elliot V, Ladipo M. One size fits all? Race, gender and body mass index among U.S. adults. J Natl Med Assoc. 2007;99:1152-8.

26. Fernald LC. Socio-economic status and body mass index in low-income Mexican adults. Soc Sci Med. 2007;64:2030-42.

27. Puone T, Steyn K, Bradshaw D, Laubscher R, Fourie J, Lambert $\mathrm{V}$, et al. Obesity in South Africa: the south african demographic and health survey. Obes Res. 2002;10:1038-48.

28. Yancev AK, Wold CM, McCarthy WJ, Weber MD, Lee B, Simon PA, et al. Physical inactivity and overweight among Los Angels County adults. Am J Prev Med. 2004;27:183-4. 
29. Cercato C, Mancini MC, Arguello AM, Passos VQ, Villares SM, Halpern A. Systemic hypertension, diabetes mellitus, and dyslipidemia in relation to body mass index: evaluation of a Brazilian population. Rev Hosp Clin Fac Med Sao Paulo. 2004;59:113-8.

30. Gus M, Moreira LB, Pimentel M, Gleisner ALM, Moraes RS, Fuchs FD. Associação entre diferentes indicadores de obesidade e prevalência da hipertensão arterial. Arq Bras Cardiol. 1998;70:111-4.

31. Bosello O, Zamboni M. Visceral obesity and metabolic syndrome. Obes Rev. 2000;1:47-56.

32. Krotkiewski M, Bjorntorp P, Sjostrom L, Smith U. Impact of obesity on metabolism in men and women. Importance of regional adipose tissue distribution. J Clin Invest. 1983;73:1150-62.

33. Pereira RA, Sichieri R, Marins VM. Razão cintura/quadril como preditor de hipertensão arterial. Cad Saúde Pública. 1999;15:333-44.

34. Kanai H, Matsuzawa Y, Kotani K, Keno Y, Kobatake T, Nagai Y, et al. Close correlation of intra-abdominal fat accumulation to hypertension in obese women. Hypertension. 1990;16:484-90.
35. Arner P. Insulin resistance in type 2 diabetes: role of fatty acids. Diabetes Metab Res Rev. 2002;18:5-9.

36. Felber JP, Golay A. Pathways from obesity to diabetes. Int J Obes Relat Metab Disord. 2002;26:39-45.

37. Frayn KN. Visceral fat and insulin resistance--causative or correlative? Br J Nutr. 2000;83:71-7.

Endereço para correspondência:

Gustavo Velásquez-Meléndez

Escola de Enfermagem da UFMG

Avenida Alfredo Balena, 190

30130-100, Belo Horizonte MG

E-mail: guveme@ufmg.br 